\title{
Tilting a ground state reactivity landscape by vibrational strong coupling
}

\author{
A. Thomas ${ }^{1 \#}$, L. Lethuillier-Karl ${ }^{1 \#}$, K. Nagarajan ${ }^{1}$, R. M. A. Vergauwe ${ }^{1}, J^{\prime}$ George ${ }^{1+}$, T. \\ Chervy $^{1 \dagger}$, A. Shalabney ${ }^{2}$, E. Devaux ${ }^{1}$, C. Genet ${ }^{1}$, J. Moran $^{1 *}$, T. W. Ebbesen ${ }^{1 *}$ \\ ${ }^{1}$ University of Strasbourg, CNRS, ISIS \& icFRC, \\ 8 allée G. Monge, 67000 Strasbourg, France. \\ ${ }^{2}$ Braude College, Snunit Street 51, Karmiel 2161002, Israel
}

*Correspondence to: ebbesen@unistra.fr; moran@unistra.fr

\#These authors contributed equally

Present Address:

Department of Chemical Sciences, Indian Institute of Science Education and research Mohali, Mohali, India.

†'Institute for Quantum Electronics, ETH Zürich, CH-8093 Zürich, Switzerland

\section{Abstract:}

Many chemical methods have been developed to favor a particular product in transformations of compounds that have two or more reactive sites. Here we explore a different approach to site-selectivity using vibrational strong coupling (VSC) between a reactant and the vacuum field of a microfluidic optical cavity. Specifically, we studied the reactivity of a compound bearing two possible silyl bond cleavage sites, $\mathrm{Si}-\mathrm{C}$ and Si-O, respectively, as a function of VSC of three distinct vibrational modes in the dark. The results show that VSC can indeed tilt the reactivity landscape to favor one product over the other. Thermodynamic parameters reveal the presence of a large activation barrier and significant changes to the activation entropy, confirming the modified chemical landscape under strong coupling.

\section{One Sentence Summary:}

The selectivity of a chemical reaction is reversed by the light-matter hybridization of its vibrational transitions to the vacuum electromagnetic field.

Over the years, synthetic chemists have refined the art of designing and developing bond-, siteand stereoselective chemical reactions using various functional moieties and catalysts. In light of the decisive nature of molecular vibrations in dictating the outcome of isomerization and other chemical processes (1-3), a physical approach based on the selective excitation of vibrational modes using strong laser fields has also been explored to impart control on chemical reactivity (47). This laser-induced mode-selective chemistry aimed to steer the system along the reaction coordinate, and appeared most straightforward for late barrier reactions (8-9). The vibrational excitation of the $\mathrm{C}-\mathrm{H}$ bond of an early barrier reaction inhibited formation of the typical product and modified the branching ratio (10), even though such an excitation was predicted to have little influence on the outcome (11). An adiabatic transfer of excitation to the product stretching mode was also observed when the localized spectator mode of the reactant was pumped in the former experiments, further indicating the complexities of the reactive landscape $(10,12)$. Although such 
mode-selective chemistry works well in the gas phase at cryogenic temperatures, intramolecular vibrational energy redistribution (IVR) limits its use for reactions in solution involving large molecules. To overcome such challenges, we started exploring an alternative approach whereby the vibrational transition of a molecule is selectively hybridized with the vacuum electromagnetic field of a cavity by means of vibrational strong coupling (VSC) (13-16). In other words, even in the dark, the zero-point energy fluctuations of the cavity mode can interact with the vibrational transition and lead to the formation of two new vibropolaritonic states (P+ and P- in Fig. 1A) that should affect the Morse potential. We predicted that this splitting could lead to changes in chemical reactivity (17) and indeed demonstrated that the rate of silyl cleavage in 1-phenyl-2-trimethylsilylacetylene is reduced by VSC of the Si-C stretching vibration (18). The reaction mechanism was also modified.

Changing the ground state reactivity landscape under such a weak field perturbation suggests that VSC could also be used to modify site-selectivity. Here in a proof-of-principle study, we demonstrate that it is indeed possible to tilt the chemical landscape of a reactant to favor one product over another.

A

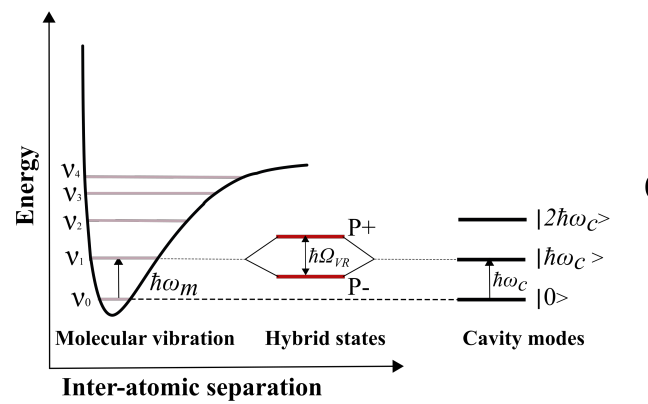

B
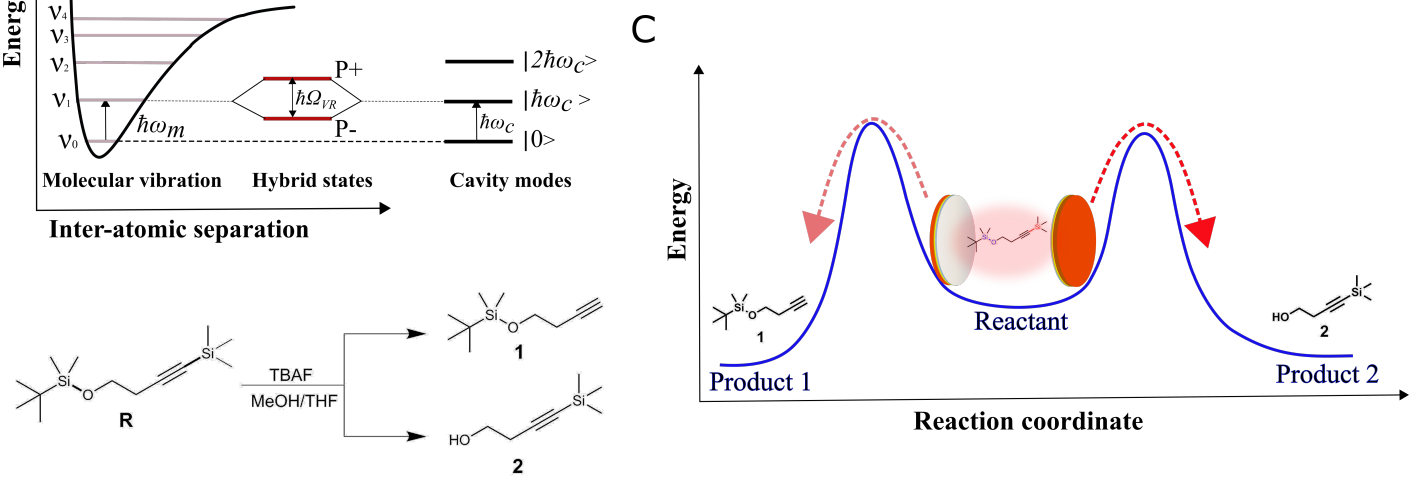

Fig. 1. Test reaction to probe the influence of VSC on site selectivity. (A) Schematic illustration of the light-matter strong coupling of a vibrational transition in resonance with Fabry-Pérot cavity mode and the subsequent formation of hybrid polaritonic states $\mathrm{P}+$ and $\mathrm{P}$ - separated by Rabi splitting energy, $\hbar \Omega_{\mathrm{R}}$. (B) The two major silyl cleavage pathways ( $\mathrm{Si}-\mathrm{C}$ scission to form $\mathbf{1}$, Si-O scission to form 2 ) for the reaction of $\mathbf{R}$ with tetrabutylammonium fluoride (TBAF) in a roomtemperature mixture of methanol and tetrahydrofuran (THF). (C) Schematic representation of competing site-selective pathways upon VSC of the reactant.

To explore the above possibilities, we synthesized the silane derivative, tert-butyldimethyl((4(trimethylsilyl)but-3-yn-1-yl)oxy)silane called $\mathbf{R}$ hereafter (Fig.1B), which incorporates two distinct sites for prospective silyl bond cleavage (See supplementary material for details and refs. 19 and 20). Nucleophilic attack by a fluoride ion on either Si atom can result in the respective cleavage of the Si-C bond, yielding product 1, or the Si-O bond, yielding product 2 (Fig. 1B). Because both products form through similar mechanistic pathways, first we explored whether the selective strong coupling of vibrational modes respectively associated with $\mathrm{Si}-\mathrm{C}$ and $\mathrm{Si}-\mathrm{O}$ have similar or different influences on the reactivity. The second and most important point of the present 
study was to ascertain whether changes in the reactivity landscape under VSC lead to siteselectivity and thereby changes to the branching ratio of the products as schematically presented in Fig.1B and C.

Light-matter hybridization is generally achieved by placing molecules in the liquid or solid phase into a Fabry-Perot optical cavity with a mode tuned in resonance with the chosen molecular transition (21). When the exchange of energy between the molecular transition and cavity mode is faster than any decay process, the system is said to be in a strong coupling regime manifested by the splitting of the molecular transition into two new states separated by a Rabi splitting energy, $\hbar \Omega_{\mathrm{R}}$, as shown schematically in Fig. 1A with VSC as a specific example. Strong coupling strength and therefore the Rabi splitting increases as the square root of the concentration of coupled molecules (21). Under such collective strong coupling conditions, the Rabi splitting can be so large that it results in significant modification of characteristic properties beyond chemical reactivity such as electrical conductivity (22), and rates of energy transfer (23-24) and reverse intersystem crossing (25). This has led to many experimental and theoretical studies of strongly coupled molecular systems (e.g. refs. 26-38). For those not familiar with light-matter strong coupling, it might be understood by analogy with the concept of a charge transfer complex. Whereas electrons are shared between two moieties in a charge transfer complex, in light-matter strong coupling, photons are shared between the cavity and the molecules. While it is well established that in the first case chemical properties change, here we demonstrate again that this is also the case for strong coupling. For a detailed introductory explanation of strong coupling of molecules, please see ref. 21.

The Fabry-Pérot cavity used in the present experiments comprised two parallel metal mirrors (Au-coated $\mathrm{ZnSe}$ ) separated by Mylar spacers of different thicknesses (6-8 $\mu \mathrm{m})$ with a hollow central channel designed to inject liquid samples (15). To ensure that the reaction dynamics were not affected by the presence of $\mathrm{Au}$ or $\mathrm{ZnSe}$, an additional $200 \mathrm{~nm}$ thick layer of glass $\left(\mathrm{SiO}_{2}\right)$ was deposited on the inner surface of each window. A homogenous solution of the starting material $\mathbf{R}(0.90 \mathrm{M})$ and tetrabutylammonium fluoride (TBAF, $0.86 \mathrm{M})$ in a $1: 1(\mathrm{v} / \mathrm{v})$ mixture of methanol and THF, optimized for strong coupling conditions by using a high concentration of $\mathbf{R}$ to achieve the highest Rabi splittings, was used as the reaction medium throughout the experiments presented here. The infrared (IR) spectrum of $\mathbf{R}$ (Fig. 2A) in this mixture recorded outside the cavity shows 
the characteristic strong bands corresponding to the $\mathrm{Si}-\mathrm{C}\left(842 \mathrm{~cm}^{-1}\right)$ and $\mathrm{Si}-\mathrm{O}\left(1110 \mathrm{~cm}^{-1}\right)$ stretching modes and the bending mode of the $\mathrm{CH}_{3}$ groups bonded to $\mathrm{Si}\left(1250 \mathrm{~cm}^{-1}\right)$.
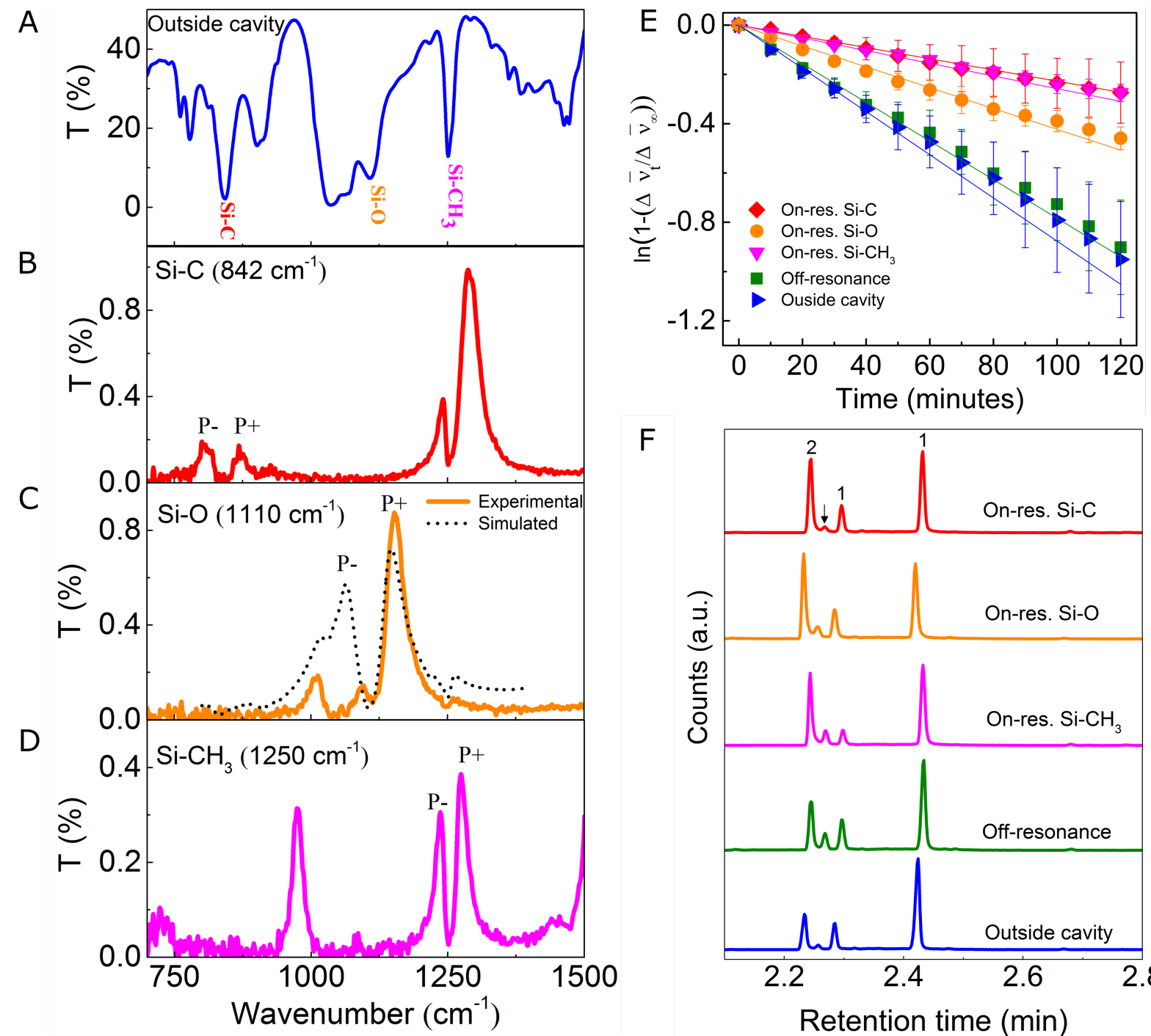

$\mathrm{F}$

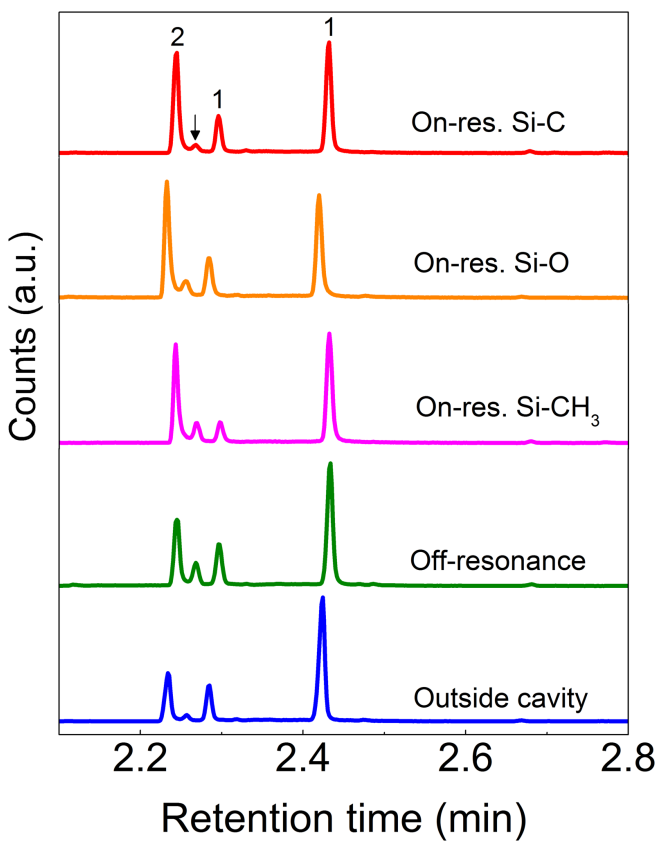

Fig. 2. VSC spectra and relative bond scission kinetics. (A) FT-IR transmission spectrum of the starting material $\mathbf{R}$ in the reaction mixture recorded in a sample cell with glass-coated $\mathrm{ZnSe}$ windows separated by the same spacer thickness as of the Fabry-Pérot cavity. (B-D) FT-IR transmission spectra showing $\mathrm{P}+$ and $\mathrm{P}$ - splitting when the three different modes are strongly coupled to the cavity: (B) the $\mathrm{Si}-\mathrm{C}$ stretching mode, (C) the $\mathrm{Si}-\mathrm{O}$ stretching mode together with a transfer matrix simulated transmission spectrum (black dotted line), and (D) $\mathrm{Si}_{-} \mathrm{CH}_{3}$ bending vibration. (E) Kinetics of the reactions in cavities tuned to be on-resonance with the Si-C stretching (red diamonds), the Si-O stretching (orange circles) and the $\mathrm{Si}-\mathrm{CH}_{3}$ bending modes (magenta triangles) together with the data for an off-resonance cavity (green squares) and outside the cavity (blue triangles), as extracted from the temporal shifts in the higher-order cavity modes. The error bars show the standard deviations for a minimum of five independent experiments for each case (F) The GC-MS chromatograms of the reactions carried out in cavities tuned to be on-resonance with Si-C stretching (red), Si-O stretching (orange) and $\mathrm{Si}-\mathrm{CH}_{3}$ bending modes (magenta), together 
with the data for an off-resonance cavity (green) and outside the cavity (blue). The peaks corresponding to $\mathrm{C}$ - and $\mathrm{O}$ - deprotected products are marked as $\mathbf{1}$ and 2, respectively. The peak indicated by the arrow is an impurity that comes from the slow degradation of the GC-MS column and it is present in all the chromatograms that we measure. These chromatograms are zoomed in on the products for clarity, the complete ones can be found in the Supplementary Materials Fig. S9.

VSC requires the cavity mode to be in resonance with a specific IR transition of $\mathbf{R}$. This was achieved through careful adjustment of the spacer thickness by tightening or loosening the screws holding the assembly together to vary the applied pressure (see Supplementary Materials for details). Injection of the homogeneous reaction mixture into the flow cell Fabry-Pérot cavity, whose second mode $(\lambda)$ was precisely tuned to $842 \mathrm{~cm}^{-1}$, resulted in the strong coupling of the Si$\mathrm{C}$ stretching transition as shown in Fig. 2B. The appearance of $\mathrm{P}+$ and $\mathrm{P}$ - peaks in the IR spectrum with a Rabi splitting energy of $70 \mathrm{~cm}^{-1}$, larger than the width of the cavity mode $\left(30 \mathrm{~cm}^{-1}\right)$ and the Si-C vibrational band $\left(30 \mathrm{~cm}^{-1}\right)$, showed that the system meets the strong coupling criteria $(21)$. Under these conditions, a largely detuned coupling was also observed between the bending mode of the $\mathrm{CH}_{3}$ groups bonded to $\mathrm{Si}\left(\mathrm{Si}-\mathrm{CH}_{3}, 1250 \mathrm{~cm}^{-1}\right)$ and the third mode of the cavity. The influence of the strongly coupled $\mathrm{Si}-\mathrm{CH}_{3}$ bending vibration on the reactivity was analyzed separately and is described later. In a different experiment, VSC of the Si-O stretching band was achieved by tuning the third mode of the cavity to $1110 \mathrm{~cm}^{-1}$ (Fig. 2C solid line). The presence of the intense and broad vibrational modes of the solvents $\left(1040-1060 \mathrm{~cm}^{-1}\right)$, splits and reduces the P- band intensity (Fig. 2C) and complicates the spectrum analysis. Therefore, the Rabi splitting energy in this case was calculated using the standard optics technique (transfer matrix simulation as explained in the Supplementary Material) shown in Fig. $2 \mathrm{C}$ as a dotted line. The extracted value of $85 \mathrm{~cm}^{-1}$ is well above the cavity and IR mode widths.

The sum rates of the two parallel silyl cleavage reactions under the influence of VSC were quantitatively determined from the $\mathrm{ln}$ plot of the temporal shift of a higher order cavity mode (from $\bar{v}_{0}$ to $\bar{v}_{\mathrm{t}}$; see Supplementary Materials for details). Similar to the earlier experiment (18), this analysis was aided by the slight change in the refractive indices of the reactant and products. The linearity of the ln plot (Fig. 2E) supported first-order kinetics, and from the slope the rate 30 constant corresponding to the disappearance of starting material was determined. The observed first-order kinetics for this bimolecular reaction is an indication of a complex reaction mechanism as documented in the literature $(39,40)$. When the $\mathrm{Si}-\mathrm{C}$ stretching bond vibration was strongly coupled, the rate was retarded (Fig. 2E, red diamond) by a factor of $3.5 \pm 0.2$ compared to the control measurements done outside the cavity (Fig. 2E, blue triangle) or inside the cavity under off-resonance conditions (Fig. 2E, green square), when the cavity was not tuned to any of the selected vibrational transitions of $\mathbf{R}$. Similarly, strong coupling to the $\mathrm{Si}-\mathrm{O}$ stretch to the cavity slowed the overall rate by a factor of $2.5 \pm 0.1$ relative to the outside cavity/off resonant conditions (orange circle, Fig. 2E). The error margin was determined from the standard deviation of a minimum of 5 experiments.

The reaction mixtures were quantitatively analyzed by gas chromatography/mass spectrometry (GC-MS) after each experiment (see Supplementary Materials for details). The presence of unreacted starting material (labeled $\mathbf{R}$ in Fig. S9 in Supplementary Material) in all the chromatograms showed that the reaction was far from completion in the $3 \mathrm{~h}$ time period and that the ratio did not change over time within the limits of the experiments. The ratios $([1] /[2])$ of the 
Si-C cleavage to Si-O cleavage product concentrations were further quantified from the GC-MS. For outside cavity and off-resonance reactions, the ratios favored $\mathrm{Si}-\mathrm{C}$ cleavage and were equivalent within experimental error (1.5 \pm 0.2 and $1.4 \pm 0.1$ respectively). A remarkable increase in the peak area of product 2 was seen when the Si-C stretching vibration was strongly coupled (red chromatogram, Fig. 2F), with a modified branching ratio [1]/[2] of $0.3 \pm 0.1$. This value takes into account the fraction of the Fabry-Perot microfluidic cavity under strong coupling. As explained in the Supplementary Material, about $60 \%$ of the cavity is under VSC, due to the deformation of the mirrors when the cavity is tuned by applying pressure with screws. The remaining $40 \%$ is detuned beyond the width of the vibrational mode and as a consequence is in the off-resonance condition. At the same time, the reaction rate is monitored through a small aperture in the central tuned area. A higher product ratio $(0.4 \pm 0.1)$ was observed when the Si-O stretching vibration was strongly coupled. Thus, VSC of the stretch vibration at either site modifies the reactive landscape in favor of Si-O cleavage over the otherwise kinetically favored Si-C cleavage.

As discussed earlier, previous mode-selective chemistry experiments have shown that spectator modes, vibrations that are not directly linked to the reaction coordinate, also play a critical role in determining reaction outcomes $(10,41)$. In the present case, the strong IR band at $1250 \mathrm{~cm}^{-1}$, arising from the bending mode of the $-\mathrm{CH}_{3}$ groups bonded to $\mathrm{Si}$ (42) can be considered a spectator vibration. However, the appearance of this IR transition at $1250 \mathrm{~cm}^{-1}$, which one would normally expect at ca. $1460 \mathrm{~cm}^{-1}$ if it is an isolated $-\mathrm{CH}_{3}$ group, already indicates that it is interacting with other vibrations associated with the Si center. By varying the spacer thickness, we achieved VSC of this bending mode alone (Fig. 2D) with a Rabi splitting energy of $43 \mathrm{~cm}^{-1}$, without simultaneously coupling either the Si-C or Si-O stretching vibrations. VSC of the 1250 $\mathrm{cm}^{-1}$ vibration reduced the overall reaction rate by a factor of $3.5 \pm 0.1$ (Fig. 2E, magenta triangle) in close similarity to the results obtained under VSC of the $\mathrm{Si}-\mathrm{C}$ stretching transition and gave rise to a product ratio $[1] /[2]$ of $0.3 \pm 0.1$ (Fig. $2 \mathrm{~F}$, magenta chromatogram). The question then naturally arises whether the VSC of any vibrational mode of $\mathbf{R}$ would lead to similar results or whether the effects are restricted to those vibrations that are associated with the reactive Si centers. To explore this question, we coupled the cavity to the C-O stretching transition (at $1045 \mathrm{~cm}^{-1}$ ) of $\mathbf{R}$, a vibration that should have little influence on the reaction from a mechanistic point of view. This mode is also embedded in the broad region (1040-1060 $\mathrm{cm}^{-1}$, see Fig. 2A) corresponding to the C-O vibrations of the solvents used (methanol and THF), and so should also serve as a probe of whether coupling to a solvent vibration influences the outcome of the reaction. We observed that neither the rate nor the product ratio was affected by VSC of this C-O transition (Fig. S10).

These experiments were completed by measuring the overall observed reaction rate $\left(k_{T R}\right)$ as a function of cavity tuning across the IR spectrum of $\mathbf{R}$ to obtain an action spectrum (Fig. 3A) which clearly confirms which vibrational modes influence the reaction under VSC. This plot reflects the precise selectivity that can be achieved by this weak field physical perturbation tool at room temperature. It should be noted that tuning the cavity so that VSC occurs at normal incidence is essential to observe the modification of chemical properties. In this condition, the system is at the minimum energy in the polaritonic state.

By combining the observed overall rate constant $\left(k_{T R}\right)$ of the parallel reaction and the product ratios $([1] /[2])$ from GC-MS measurements, we estimated the individual rate constants corresponding to the $\mathrm{Si}-\mathrm{C}\left(k_{1}\right)$ and $\mathrm{Si}-\mathrm{O}\left(k_{2}\right)$ bond scissions. From the data, the respective product yields were calculated. The yields $(\phi)$ of products $\mathbf{1}$ and $\mathbf{2}$ in and out of strong coupling for various 
coupling conditions are shown in Fig. 3B. Outside the cavity and in off-resonance conditions, the $k_{1}$ dominates over $k_{2}$ resulting in preferential formation of product 1 (Fig. 3B, violet diamond). VSC reverses the selectivity, leading to an excess formation of product 2 (Fig. 3B, pink squares). This is again a direct proof-of-principle that the chemical landscape can be tilted by targeting the three vibrational modes associated with the reaction centers. Although they do appear similar in this study, the rates and the yields vary by as much as $25 \%$ to $40 \%$ with VSC between these modes. The values of both $k_{1}$ and $k_{2}$ under strong coupling conditions are lower than those observed outside the cavity indicating that a higher activation energy is required for the reaction under VSC. Products $\mathbf{1}$ and $\mathbf{2}$ undergo further silyl bond cleavage, though the subsequent reactions appear to be much slower, resulting in the accumulation of $\mathbf{1}$ and $\mathbf{2}$ in the GC-MS analysis. These downstream reactions impart a small error in the yields but has no consequence on the overall results.
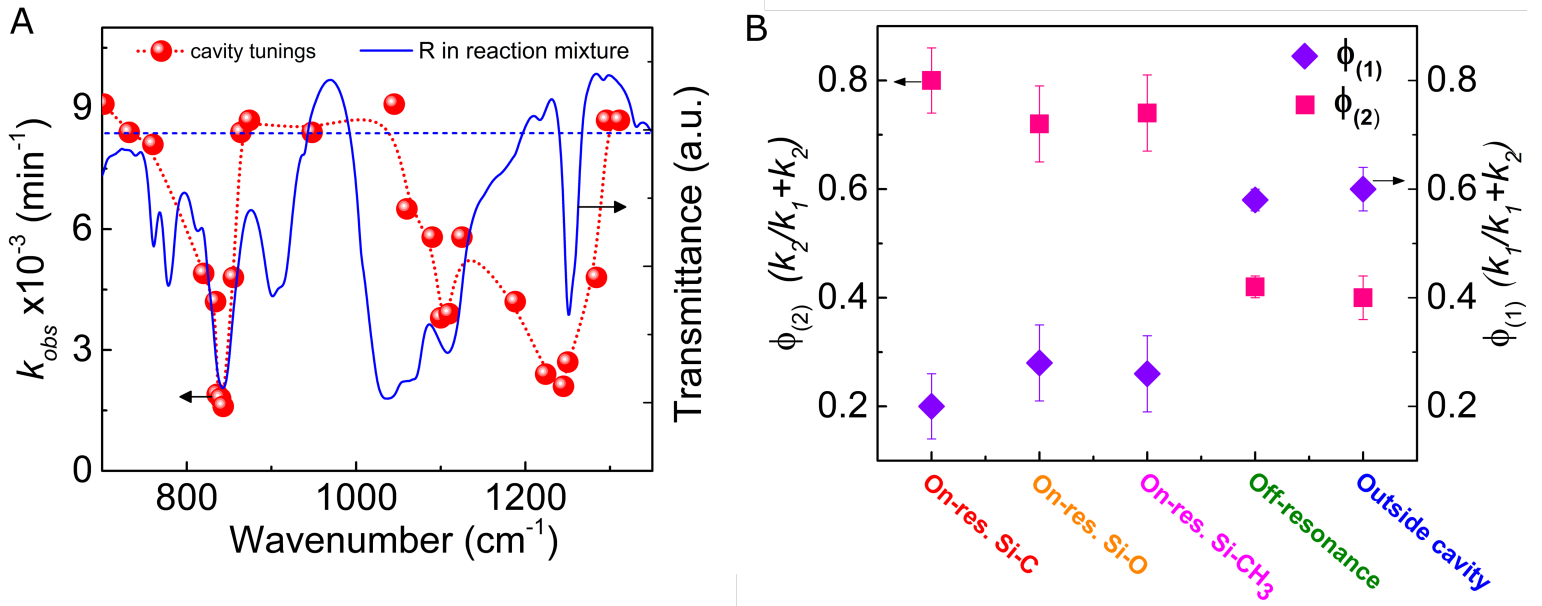

Fig.3. Relative VSC influence across the vibrational spectrum. (A) The overall reaction rate as a function of cavity tuning for reactions inside the cavity (red spheres). The blue solid line shows the IR absorption spectrum of $\mathbf{R}$ in the reaction medium. The red dotted line connecting the spheres is a guide for the eye. The blue dashed line represents the average rate of the reaction outside the cavity. (B) Plot showing the yields of product $\mathbf{1}\left(\phi_{1}\right.$, violet diamonds) and $\mathbf{2}$ ( $\phi_{2}$, pink squares) under VSC of various vibrational modes of $\mathbf{R}$, together with the off-resonance and outside cavity conditions. The error margin was determined from the standard deviation of a minimum of 5 experiments in each case.

The thermodynamics of the two bond breaking reactions leading to products $\mathbf{1}$ and $\mathbf{2}$ were determined by measuring the rates as a function of temperature under VSC of the Si-C and Si-O stretching vibrations as well as outside the cavity. Using transition state theory, the free energy of activation $\left(\Delta \mathrm{H}^{\ddagger}\right)$ and the entropy of activation $\left(\Delta \mathrm{S}^{\ddagger}\right)$ were determined from the slope and intercept of the plot of $\ln (k)$ against the inverse of temperature (Fig. 4). The table 1 summarizes the results extracted from the plots of Figure 4. It shows that the thermodynamic parameters are significantly changed under VSC for both products but lead to different values for products $\mathbf{1}$ and $\mathbf{2}$. What is surprising is that the new values of $\Delta \mathrm{H}^{*}$ and $\Delta \mathrm{S}^{*}$ appear similar whether one couples the Si-C or 
the Si-O stretching vibrations. This might be fortuitous for the specific molecule under study. Interestingly, it has been shown that the presence of leaving groups far away can change desilylation rates (43).
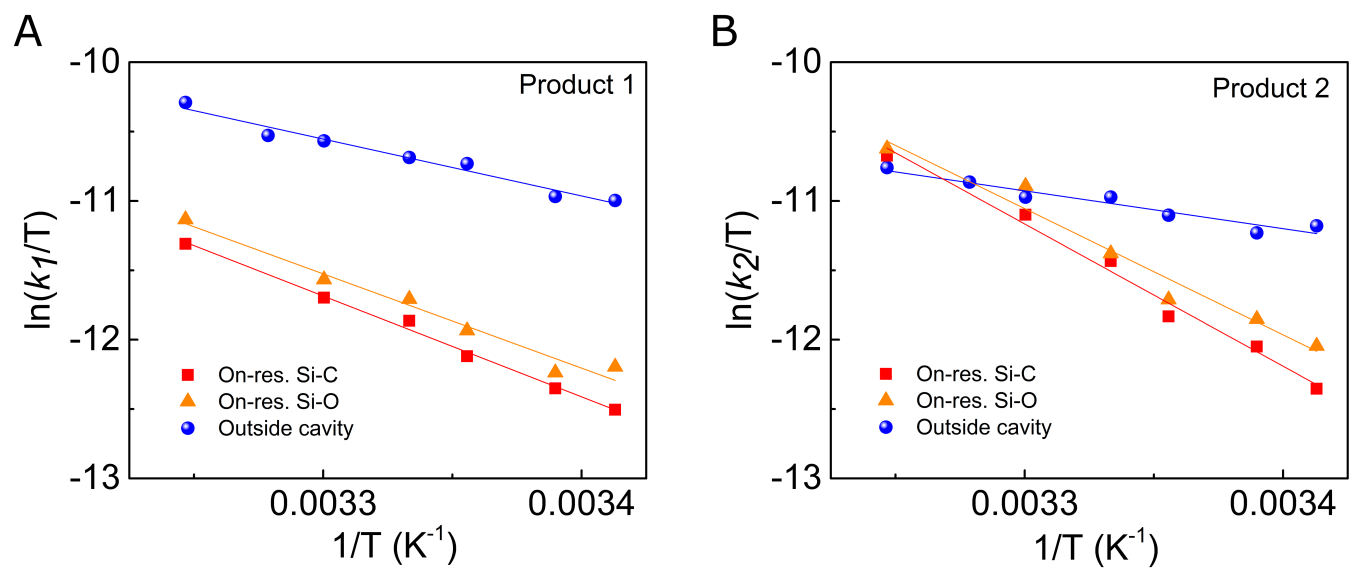

Fig. 4. Eyring plots showing the reaction rate for the formation of products as a function of temperature between 20 and $35^{\circ} \mathrm{C}$. (A) formation of product 1, (B) formation of product 2. Reactions inside the cavities tuned to be on-resonance with $\mathrm{Si}-\mathrm{C}$ (red squares) or Si-O (orange triangles) stretching modes are compared to reactions outside the cavity (blue spheres). The data were fitted with a least-square method and the quality of the fit can be assessed from R-square values which are $\geq 0.95$.

Table 1.

\begin{tabular}{cccc}
\hline Products & Experiment & $\begin{array}{c}\Delta \mathbf{H}^{*} \\
(\mathbf{k J m o l}\end{array}$ & $\begin{array}{c}\Delta \mathbf{S}^{\mathbf{1}} \mathbf{)} \\
\left(\mathbf{J m o l}^{\mathbf{- 1}} \mathbf{K}^{\mathbf{- 1}} \mathbf{)}\right.\end{array}$ \\
\hline Product 1 & Outside cavity & $34 \pm 3$ & $-173 \pm 11$ \\
& On-res. Si-C & $60 \pm 2$ & $-95 \pm 7$ \\
& On-res. Si-O & $57 \pm 5$ & $-106 \pm 17$ \\
Product 2 & Outside cavity & $23 \pm 3$ & $-214 \pm 8$ \\
& On-res. Si-C & $85 \pm 5$ & $-6 \pm 17$ \\
& On-res. Si-O & $76 \pm 7$ & $-39 \pm 24$ \\
\hline
\end{tabular}

The above results show that VSC can modify the chemical landscape, and the associated $\Delta \mathrm{H}^{\ddagger}$ and $\Delta \mathrm{S}^{\ddagger}$, in such a way that it tilts the landscape from one product to another. An interesting aspect of chemical reactions under VSC so far has been the high activation barrier seen towards 
the breaking of Si-C and Si-O bonds. Because a typical fluoride-induced silyl cleavage is known to proceed through an associative mechanism involving a pentacoordinate intermediate (39), a strained transition state with large negative $\Delta \mathrm{S}^{*}$ is expected as observed outside the cavity. The observed sharp decrease of $\Delta \mathrm{S}^{*}$ to a lower value under VSC implies that the transition state is strongly modified, particularly the one leading to product 2 . The high activation energy and the less negative $\Delta \mathrm{S}^{*}$ clearly indicate that the reaction switched to a dissociative mechanism under $\mathrm{VSC}$, and also suggest that the transition state is product-like as in the case of late barrier reactions. One potential explanation for the apparent change in mechanism is that VSC suppresses typical associative pathways for $\mathrm{Si}-\mathrm{C}$ and $\mathrm{Si}-\mathrm{O}$ bond cleavage, allowing otherwise slower dissociative 10 pathways to dominate. The stronger change under VSC for the dissociative pathway leading to 2 compared to that leading to $\mathbf{1}$ could therefore be rationalized since the former involves a better leaving group.

Barrier increases have also been seen in earlier experiments, whether occurring in the ground state (18) or via the excited state under electronic strong coupling such as in the photoisomerization reaction of merocyanine (17). For the latter case, recent theoretical studies show that the collective response of molecules plays a role in widening the barrier at the conical intersection and thereby preventing the isomerization $(31,32)$. However, such findings cannot yet be generalized since a recent study of hydrolysis reactions under VSC show huge rate accelerations (up to $10^{4}$ ) (44). More experiments and theoretical studies are required to be able to extract general features and understand the effects of VSC on different types of reactions.

In conclusion, this proof-of-principle study shows that it is possible to control reaction selectivity through VSC. Strong coupling is dependent on the orientation of the transition dipole moment relative to the electric field of the cavity mode. Here the molecules were randomly oriented and the electric field is not uniform in the cavity, i.e. there are nodes where the field is essentially null. Hence, the five-fold increase in selectivity under VSC reported here is probably much larger if corrected for such features. For instance, experiments could be done in a tubular cavity to ensure a larger fraction of coupled molecules. Therefore, the selectivity displayed by VSC in the current experiment shows the remarkable potential of light-matter strong coupling for enhancing site selectivity of organic and inorganic chemical reactions. More reactions must be

30 studied to evaluate the generality of this phenomenon and to see to what extent the selectivity can be enhanced at a given site. As more and more studies show, this weak field room temperature method has the potential to become an everyday tool for chemists to physically control chemical reactivity without catalysts, pre-functionalization or chemical changes to the reaction conditions.

\section{References and Notes}

1. R. T. Hall, G. C. Pimentel, J. Chem. Phys. 38, 1889-1897 (1963).

2. Q. Wang, R. W. Schoenlein, L. A. Peteanu, R. A. Mathies, C. V. Shank, Science. 266, 422424 (1994).

3. M. Delor et al., Science. 346, 1492-1495 (2014). 
4. H. Frei, L. Fredin, G. C. Pimentel, J. Chem. Phys. 74, 397-411 (1981).

5. A. Sinha, M. C. Hsiao, F. F. Crim, J. Chem. Phys. 94, 4928-4935 (1991).

6. R. N. Zare, Science. 279, 1875-1879 (1998).

7. F. F. Crim, Acc. Chem. Res. 32, 877-884 (1999).

8. S. Yan, Y.-T. Wu, B. Zhang, X.-F. Yue, K. Liu, Science. 316, 1723-1726 (2007).

9. D. R. Killelea, V. L. Campbell, N. S. Shuman, A. L. Utz, Science. 319, 790-793 (2008).

10. W. Zhang, H. Kawamata, K. Liu, Science. 325, 303-306 (2009).

11. J. C. Polanyi, Acc. Chem. Res. 5, 161-168 (1972).

12. K. Liu, Annu. Rev. Phys. Chem. 67, 91-111 (2016).

13. A. Shalabney et al., Nat. Commun. 6, 5981 (2015).

14. J. P. Long, B. S. Simpkins, ACS Photonics. 2, 130-136 (2015).

15. J. George et al., Phys. Rev. Lett. 117, 153601 (2016).

16. R. M. A. Vergauwe et al., J. Phys. Chem. Lett. 7, 4159-4164 (2016).

17. J. A. Hutchison, T. Schwartz, C. Genet, E. Devaux, T. W. Ebbesen, Angew. Chem. Int. Ed. 51, 1592-1596 (2012).

18. A. Thomas et al., Angew. Chem. Int. Ed. 55, 11462-11466 (2016).

19. R. J. Rahaim, J. T. Shaw, J. Org. Chem. 73, 2912-2915 (2008).

20. S. V. Shelke et al., Angew. Chem. Int. Ed. 49, 5721-5725 (2010).

21. T. W. Ebbesen, Acc. Chem. Res. 49, 2403-2412 (2016).

22. E. Orgiu et al., Nat. Mater. 14, 1123-1129 (2015).

23. X. Zhong et al., Angew. Chem. Int. Ed. 56, 9034-9038 (2017).

24. C. Gonzalez-Ballestreo, J. Feist, E. Moreno, F.J. Garcia-Vidal, Phys. Rev. B 92, 121402 (2015).

25. K. Stranius, M. Hertzog, K. Börjesson, Nat. Commun. 9, 2273 (2018).

26. S. Wang, T. Chervy, J. George, J.M. Hutchison, C. Genet, T.W. Ebbesen, J. Phys. Chem. Lett. 5, 1433-1439 (2014).

27. S. Gambino et al., ACS Photonics. 1, 1042-1048 (2014).

28. W. Ahn, I. Vurgaftman, A. D. Dunkelberger, J. C. Owrutsky, B. S. Simpkins, ACS Photonics. 5, 158-166 (2018).

29. M. Hertzog et al., Chem. Eur. J. 23, 18166-18170 (2017). 
30. O. Kapon, R. Yitzhari, A. Palatnik, Y. R. Tischler, J. Phys. Chem. C. 121, 18845-18853 (2017).

31. J. Galego, F. J. Garcia-Vidal, J. Feist, Nat. Commun. 7, 13841 (2016).

32. J. Galego, F. J. Garcia-Vidal, J. Feist, Phys. Rev. Lett. 119, 136001 (2017).

33. V. F. Crum, S. R. Casey, J. R. Sparks, Phys. Chem. Chem. Phys. 20, 850-857 (2018).

34. G. G. Rozenman, K. Akulov, A. Golombek, T. Schwartz, ACS Photonics. 5, 105-110 (2018).

35. D. G. Baranov, M. Wersäll, J. Cuadra, T. J. Antosiewicz, T. Shegai, ACS Photonics. 5, 2442 (2018).

36. F. Herrera, F. C. Spano, Phys. Rev. Lett. 116, 238301 (2016).

37. L. A. Martínez-Martínez, R. F. Ribeiro, J. Campos-González-Angulo, J. Yuen-Zhou, ACS Photonics. 5, 167-176 (2018).

38. J. Flick, C. Schäfer, M. Ruggenthaler, H. Appel, A. Rubio, ACS Photonics. 5, 992-1005 (2018).

39. A. R. Bassindale, P. G. Taylor, "Reaction mechanisms of nucleophilic attack at silicon" in Organic Silicon Compounds (Wiley-Blackwell, 2004), ch.13, pp. 839-892.

40. A. M. DiLauro, W. Seo, S. T. Phillips, J. Org. Chem. 76, 7352-7358 (2011).

41. G. C. Schatz, M. C. Colton, J. L. Grant, J. Phys. Chem. 88, 2971-2977 (1984).

42. A. Grill, D. A. Neumayer, J. Appl. Phys. 94, 6697-6707 (2003).

43. P. M. F. M. Bastiaansen, R. V. A. Orru, J. B. P. A. Wijnberg, A. de Groot, J. Org. Chem. 60, 6154-6158 (1995).

44. H. Hiura, A. Shalabney, J. George, (2018), doi:10.26434/chemrxiv.7234721.v2.

\section{Acknowledgments:}

Funding: We acknowledge support of the International Center for Frontier Research in Chemistry (icFRC, Strasbourg), the ANR Equipex Union (ANR-10-EQPX-52-01), the Labex NIE projects (ANR-11-LABX-0058 NIE) and CSC (ANR-10-LABX-0026 CSC) within the 


\section{Science}

MlaAAS

Investissement d'Avenir program ANR-10-IDEX-0002-02. TWE also acknowledges the support of the ERC (project no 788482 MOLUSC).

Author contributions: AT and LLK took the lead in all the experiments, assisted by the KN, RMAW, JG , TC, AS and ED. CG provided theoretical insight and JM and TWE conceived and oversaw the experiments.

Competing interests: Authors declare no competing interests

Data and materials availability: All data are available in the main text or the supplementary materials.

\section{Supplementary Materials:}

10 Materials and Methods

Figures S1 to S10 


\section{Supplementary Materials for}

\section{Tilting a ground state reactivity landscape by vibrational strong coupling}

A. Thomas ${ }^{1 \#}$, L. Lethuillier-Karl ${ }^{1 \#}$, K. Nagarajan ${ }^{1}$, R. M. A. Vergauwe ${ }^{1}$, J. George $^{1 \dagger}$, T. Chervy $^{1 \dagger}$, A. Shalabney ${ }^{2}$, E. Devaux ${ }^{1}$, C. Genet ${ }^{1}$, J. Moran $^{1 *}$, T. W. Ebbesen ${ }^{1 *}$

${ }^{1}$ University of Strasbourg, CNRS, ISIS \& icFRC, 8 allée G. Monge, 67000 Strasbourg, France.

${ }^{2}$ Braude College, Snunit Street 51, Karmiel 2161002, Israel

*Correspondence to: ebbesen@unistra.fr; moran@unistra.fr

\#These authors contributed equally

Present Address:

Department of Chemical Sciences, Indian Institute of Science Education and research Mohali, Mohali, India.

$15 \dagger$ Institute of Quantum Electronics, ETH Zürich, CH-8093 Zürich, Switzerland.

This PDF file includes:

Materials and Methods

Figs. S1 to S10 


\section{Materials and Methods}

Unless otherwise noted, all reagents and solvents were purchased from commercial suppliers and used without further purification.

The IR transmission spectra of the Fabry-Pérot cavities and the 'outside the cavity' cells were acquired by standard FT-IR (Fourier transform infrared) spectrometer (Bruker Vertex70) in transmission mode. The transmission was recorded by DTGS (deuterated triglycine sulfate) detector with $2 \mathrm{~cm}^{-1}$ resolution over 64 scans.

GC-MS analysis was performed on a GC System 7820A (G4320) connected to a MSD block 5977E (G7036A), using Agilent High Resolution Gas Chromatography Column: PN 19091S - 433UI, HP - 5MS UI, 28m×0.250 mm, 0.25 Micron, SN USD 489634H. All samples were prepared in 2-propanol (200 $\mu \mathrm{L}$ sample volume). The analysis was carried out on a splitless $1 \mu \mathrm{L}$ injection volume with an injection port temperature $250^{\circ} \mathrm{C}$. Column oven temperature program was as follows: $60{ }^{\circ} \mathrm{C}$ for $1 \mathrm{~min}$, ramped at $30^{\circ} \mathrm{C} \mathrm{min}-1$ to $310^{\circ} \mathrm{C}$ with 3 min hold, with a total running time of $12.33 \mathrm{~min}$. The mass spectrometer was turned on after 2 min and was operated at the electron ionization mode with quadrupole temperature of $150{ }^{\circ} \mathrm{C}$. Data acquisition was performed in the full-scan mode (50-500). Hydrogen (99.999\% purity) was used as carrier gas at a constant flow rate of $1.5 \mathrm{~mL} \mathrm{~min}^{-1}$.

${ }^{1} \mathrm{H}$ NMR spectrum of starting material $\mathbf{R}$ in $\mathrm{CDCl}_{3}$ was recorded on a Bruker Avance 400 $(400 \mathrm{MHz})$ spectrometer at ambient temperature and is reported in ppm scale using the residual solvent resonance as the internal reference $\left(\mathrm{CHCl}_{3}\right.$ at $\left.7.26 \mathrm{ppm}\right)$. Coupling constants $J$ are reported in $\mathrm{Hz}$, as determined using the software (NMR Notebook v2.80). ${ }^{13} \mathrm{C}$ NMR spectrum was recorded on a Bruker Avance $400(100 \mathrm{MHz})$ spectrometer and is reported in ppm using the residual solvent resonance as the internal standard $\left(\mathrm{CDCl}_{3}\right.$ at $\left.77.0 \mathrm{ppm}\right)$.

\section{Transfer-matrix simulation}

The fitting of experimental data has been done thanks to a Matlab code based on the TMatrix method, that allows to simulate the transmission spectra of multilayered samples. To do this calculation, one needs to define precisely the refractive index for each layer. For the $\mathrm{ZnSe}$ windows of the cell and the protective $\mathrm{SiO}_{2}$ layers, the refractive indexes were both calculated from experimental transmission data of those materials. The refractive index of the gold mirrors is 
calculated from the reported polynomial expression (46), with a correction in the damping factor to take into account their very small thickness. Finally, the refractive index of the material itself was determined by fitting the experimental absorption of the material measured in ATR and measured in a cell, to define it as rigorously as possible, in particular because the presence of solvents in the cell affects the Si-O bond absorption region.

\section{Synthesis of tert-butyldimethyl((4-(trimethylsilyl)but-3-yn-1-yl)oxy)silane (R)}

A reported procedure was followed to prepare the starting material $\mathbf{R}$. To a stirred solution of 4-(trimethylsilyl)but-3-yn-1-ol $(3.50 \mathrm{mmol}, 0.585 \mathrm{~mL})$ in DMF $(5 \mathrm{~mL})$ at $0{ }^{\circ} \mathrm{C}$ were added imidazole (8.75 mmol, $0.596 \mathrm{~g})$ and tert-butylchlorodimethylsilane (4.38 mmol, $0.658 \mathrm{mg})$. The mixture was allowed to warm to room temperature and was stirred for 24 hours. The reaction was quenched by the addition of aq. saturated $\mathrm{NH}_{4} \mathrm{Cl}(15 \mathrm{~mL})$. The organic layer was collected and extracted with diethyl ether through repeated washings. The combined organic fractions were then washed with water, dried over $\mathrm{Na}_{2} \mathrm{SO}_{4}$ and concentrated under reduced pressure to give the crude product as a pale yellow liquid. The crude product was purified by column chromatography on silica using a mixture of ethyl acetate and petroleum ether $(1: 9 ; \mathrm{v} / \mathrm{v})$ as the eluent to give the pure product $\mathbf{R}(0.754 \mathrm{~g} ; 84 \%)$ as a colourless liquid.

${ }^{1} \mathbf{H}$ NMR (400 MHz, $\left.\mathrm{CDCl}_{3}\right) \delta 3.72(\mathrm{t}, J=7.0 \mathrm{~Hz}, 2 \mathrm{H}, \mathbf{3}), 2.44(\mathrm{t}, \mathrm{J}=7.0 \mathrm{~Hz}, 2 \mathrm{H}, 4), 0.90$ (s, 9H, 1), $0.14(\mathrm{~s}, 9 \mathrm{H}, 5), 0.08(\mathrm{~s}, 6 \mathrm{H}, 2)$

${ }^{13} \mathbf{C}$ NMR $\left(101 \mathrm{MHz}, \mathrm{CDCl}_{3}\right) \delta 104.29(\mathbf{F}), 85.61(\mathbf{G}), 61.86(\mathbf{D}), 25.93(\mathbf{A}), 24.31$ (B), $18.40(\mathbf{E}), 0.12(\mathbf{H}),-5.22(\mathbf{C})$ 


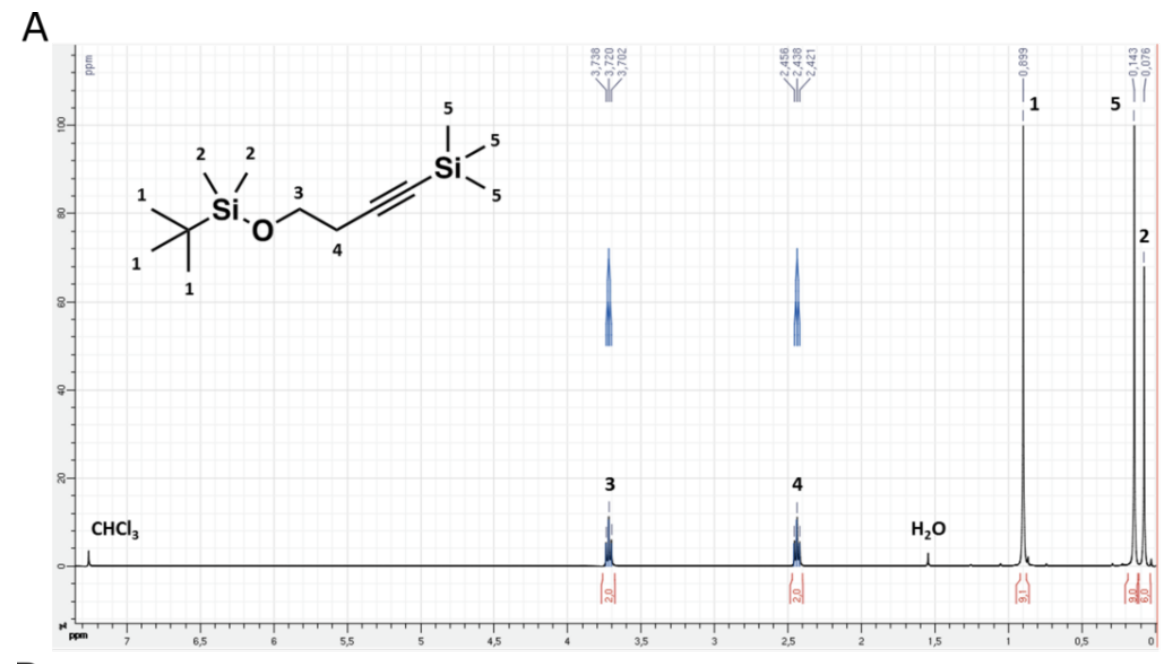

B

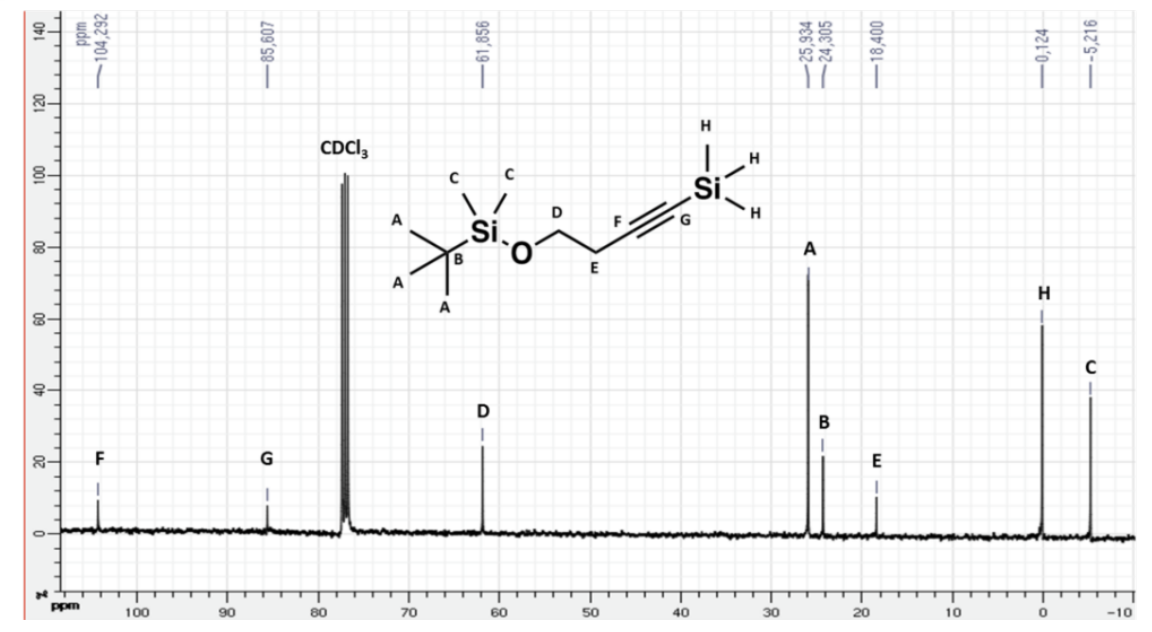

Fig. S1. (A) shows the ${ }^{1} \mathrm{H}$ NMR spectrum of the reactant $\mathbf{R}$ with its various ${ }^{1} \mathrm{H}$ signals labelled from 1 to 5 on the schematic structure of $\mathbf{R}$ given in the inset. (B) Shows the ${ }^{13} \mathrm{C}$ NMR spectrum with its various ${ }^{13} \mathrm{C}$ signals marked from $\mathrm{A}$ to $\mathrm{H}$. 


\section{FT-IR characterization of starting material, solvents and products}
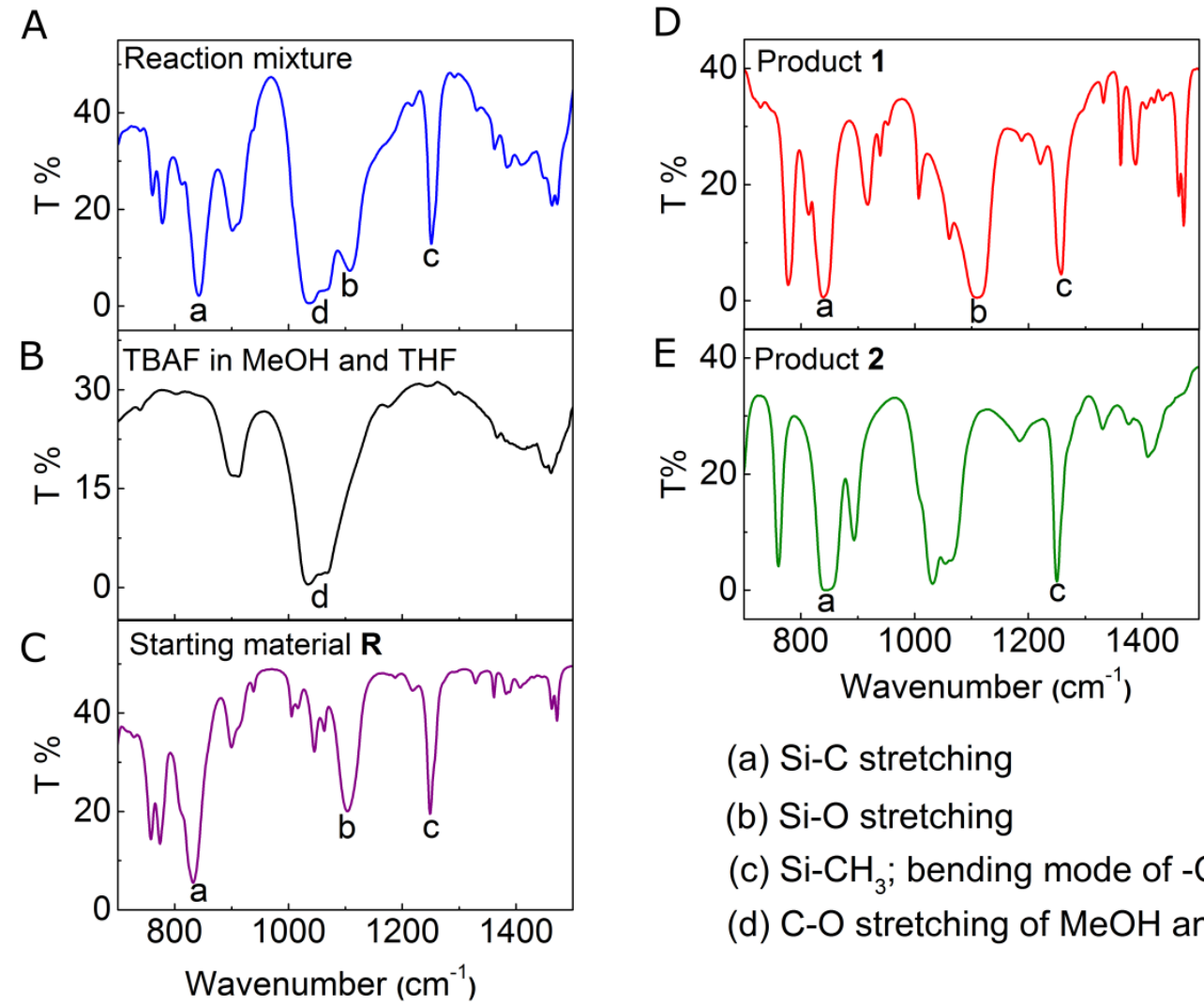

(a) Si-C stretching

(b) Si-O stretching

(c) $\mathrm{Si}-\mathrm{CH}_{3}$; bending mode of $-\mathrm{CH}_{3}$

(d) $\mathrm{C}-\mathrm{O}$ stretching of $\mathrm{MeOH}$ and THF

Fig. S2. FT-IR spectrum of the reaction mixture containing $\mathbf{R}(\mathrm{A})$, solution of TBAF in methanol $(\mathrm{MeOH})$ and THF (B), neat starting material $\mathbf{R}(\mathrm{C})$, neat product $\mathbf{1}(\mathrm{D})$ and neat product $\mathbf{2}(\mathrm{E})$. The spectrum is limited to the region of interest of the present study. The vibrational modes that are of interest in the present study are marked from a-d. Neat products were obtained from commercial sources.

\section{Preparation of the Fabry-Pérot cavity}

The IR cell (demountable flow cell) compatible for temperature controlled measurements was purchased from Specac. The $\mathrm{ZnSe}(2 \mathrm{~mm})$ were used as the IR-transmitting window substrates. The metal mirrors for the Fabry-Pérot cavity were prepared by evaporating $\mathrm{Au}(10 \mathrm{~nm})$ on to the $\mathrm{ZnSe}$ windows. To ensure chemical insulation during the reaction from both the metal and $\mathrm{ZnSe}$, each windows were coated with $200 \mathrm{~nm}$ of glass. To obtain the Fabry-Perot cavity, the 
Au coated windows were placed in the IR cell, sandwiched by Mylar spacers and tightened with the screws. To prepare the different on- and off-resonance cavities in the present experiments, the thickness of the Mylar spacers used were varied in the range of 6-12 $\mu \mathrm{m}$. The area of the cavity is $1 \mathrm{~cm} \times 2.5 \mathrm{~cm}$. The tuned part is typically $60 \%$ of this cavity area because we are using 4 screws to tune the cavity and this puts pressure on the flat windows and slightly deforms them. We check this aspect systematically, by taking the spectrum of the cavity at different points using a small aperture of $2 \mathrm{~mm}$ in diameter. The reaction is monitored through a tuned area of the cavity using an aperture of $5 \mathrm{~mm}$ in diameter. To measure the absolute yields of products we correct for the tuned area.

For reference experiments, mentioned as outside the cavity, the liquid cells were prepared in a similar manner as explained above but replacing the metal mirror with glass $(200 \mathrm{~nm})$ coated ZnSe windows.

\section{Tuning the Fabry-Perot cavities:}

Once an air-filled Fabry-Perot cavity (hereafter referred as "empty" cavity) is prepared as mentioned above, such an "empty" cavity of length L, will show a transmission spectrum with a series of resonances. The frequency of these modes, which are multiples of the fundamental cavity mode, in wavenumber units is given by the equation

$$
\bar{v}_{\left(\mathrm{cm}^{-1}\right)}=\frac{10^{4} \mathrm{~m}}{2 n L}
$$

where $\mathrm{m}$ is the mode order, $\mathrm{n}$ is the refractive index of the medium and $\mathrm{L}$ is the thickness of the cavity in micrometer. The spacing between each mode, called the free spectral range (FSR), gives the frequency corresponding to the fundamental mode. For a given spacer thickness and metal mirrors, the FSR of such an "empty" cavity will be a constant and the injection of the sample will further change the cavity mode spacing as a function of the refractive index $\mathrm{n}$ of the injected sample. As a general procedure, to prepare an on-resonance cavity with respect to a given vibrational transition, we first calculate the refractive index $\mathrm{n}$ of the medium to be injected. Subsequently, the "empty" cavity is tuned to get the required the FSR, such that when the sample is injected, one of the cavity mode is in resonance with the chosen vibrational transition. For 
example, in the present experiments, to make an on-resonance cavity with Si-C stretching transition at $842 \mathrm{~cm}^{-1}$, we first tuned our "empty" cavity to attain the FSR of $586 \mathrm{~cm}^{-1}$ as shown in Fig. S3A. Therefore the injection of our reaction mixture, whose $\mathrm{n}$ is 1.395 , will modify the FSR to $420 \mathrm{~cm}^{-1}$ and result in the strong coupling of the Si-C stretching transition with the second mode of the cavity as shown in Fig. S3B.

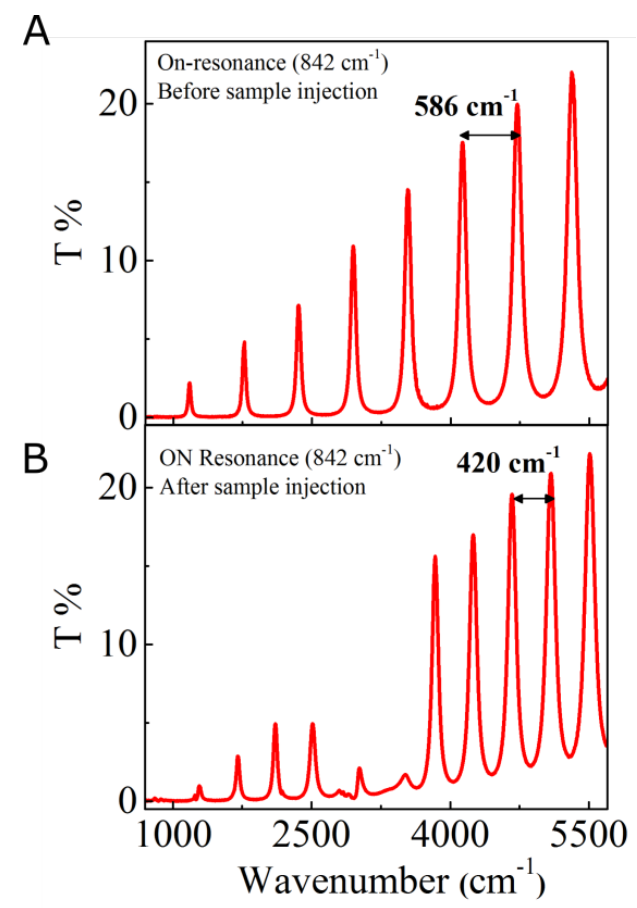

Fig. S3. Measured optical resonances of a microfluidic Fabry-Pérot cavity of length $8.5 \mu \mathrm{m}$ before $(\mathrm{A})$ and after $(\mathrm{B})$ the injection of reaction mixture.

\section{Preparation of the reaction mixture and injection into the Fabry-Perot cavities}

Once the desired "empty" cavity was obtained, the liquid sample cell was inserted in the variable temperature mount and was kept for 2 hours to stabilise and equilibrate to the experimental temperature $\left(25^{\circ} \mathrm{C}\right.$, except if noted otherwise)

The sample injection into the cavities and liquid cells were done as follows. A stock solution of TBAF (3 M) was prepared using a mixture of $\mathrm{MeOH}$ and THF in 1:1 ratio (v/v). For the reaction medium, the starting material $\mathbf{R}(0.31 \mathrm{mmol}, 100 \mu \mathrm{L})$ was mixed $\mathrm{MeOH}(75 \mu \mathrm{L})$ and THF $(75 \mu \mathrm{L})$ in a $5 \mathrm{~mL}$ glass vial followed by the addition of TBAF stock solution $(100 \mu \mathrm{L})$. The mixture was shaken to obtain a homogeneous solution and was then directly injected into the microfluidic Fabry-Perot cavity making sure that the cavity is filled without any air-bubbles. 


\section{Rate of the reaction}

The rate of the reaction was calculated from the shift in the higher order cavity mode due to a change in refractive index during the reaction (Eq. S1). The reactant and the products have slightly different reactive indices (1.39 versus 1.43 for product 1 and 1.44 for product 2 ) resulting in a change of the background refractive index ( $n$ ) of the reactive medium as the reaction progresses. Because the product ratio is constant, monitoring this shift allows one to extract the rate of the reaction as explained below. The use of $\mathrm{ZnSe}$ windows for making the sample cells for outside the cavity experiments resulted in a very weak interference fringes as shown in Fig. S4C. This is because of the relatively large difference between the $\mathrm{n}$ of $\mathrm{ZnSe}$ and the reactive medium. These weak interference fringes also shifted in the same was as that of the cavity mode, indicating the progress of the reaction.
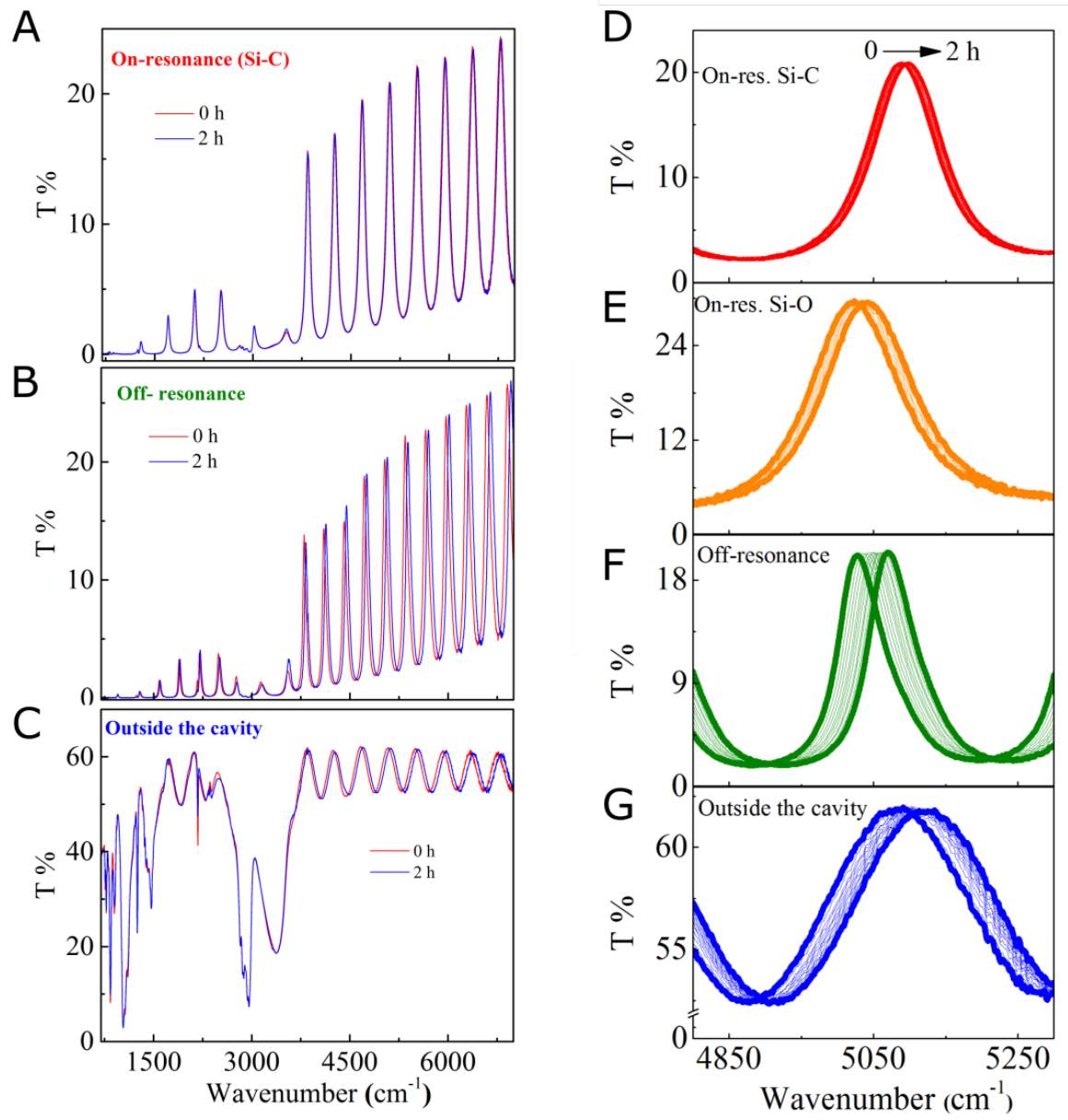
Fig. S4. FT-IR transmission spectrum of the on-resonance cavity (A), off-resonance cavity (B), and outside the cavity $(\mathrm{C})$ immediately after injection of the reaction mixture (red curve) and after $2 \mathrm{~h}$ (blue curve). (D-G) Shows the temporal shift of the higher cavity order mode during the reaction (0 to $2 \mathrm{~h}$ ) for cavities on-resonance with the $\mathrm{Si}-\mathrm{C}$ (D) and the Si-O stretching mode (E), offresonance cavity $(\mathrm{F})$ and outside the cavity $(\mathrm{G})$.

To calculate the rate of the reaction, the temporal shift $\left(\Delta \bar{v}_{t}\right)$ of a higher order cavity mode

peak for all on- as well as off-resonance experiments with respect to the starting value $\left(\bar{v}_{0}\right)$ was recorded. For experiments outside the cavity, the peak of a weak interference fringe was considered. For all these experiments a mode at $\sim 5000 \mathrm{~cm}^{-1}$, well isolated from the molecular vibrational modes were monitored as shown in Fig.S4. The maximum shift $\left(\Delta \bar{v}_{\infty}\right)$ corresponding to the completion of the reaction was determined separately from an outside the cavity experiment. The $\ln$ plot of the temporal shift $\left(\ln \left(1-\Delta \bar{v}_{t} / \Delta \bar{v}_{\infty}\right)\right)$ gave a straight line, indicating a pseudo first-order reaction and from the slope the rate constant for the total reaction $\left(k_{T R}\right)$ was calculated.

\section{Stability check of the strongly coupled starting material}

To check if the starting material alone could induce temporal shift of a higher order mode cavity, a control experiment was carried out. The staring material $\mathbf{R}$ was injected to a cavity tuned to be in resonance with the $\mathrm{Si}-\mathrm{CH}_{3}$ vibrational transition $\left(1250 \mathrm{~cm}^{-1}\right)$ and the transmission spectrum was recorded at different time intervals (Fig.S5). No shift of the cavity modes were observed even after $5 \mathrm{~h}$.
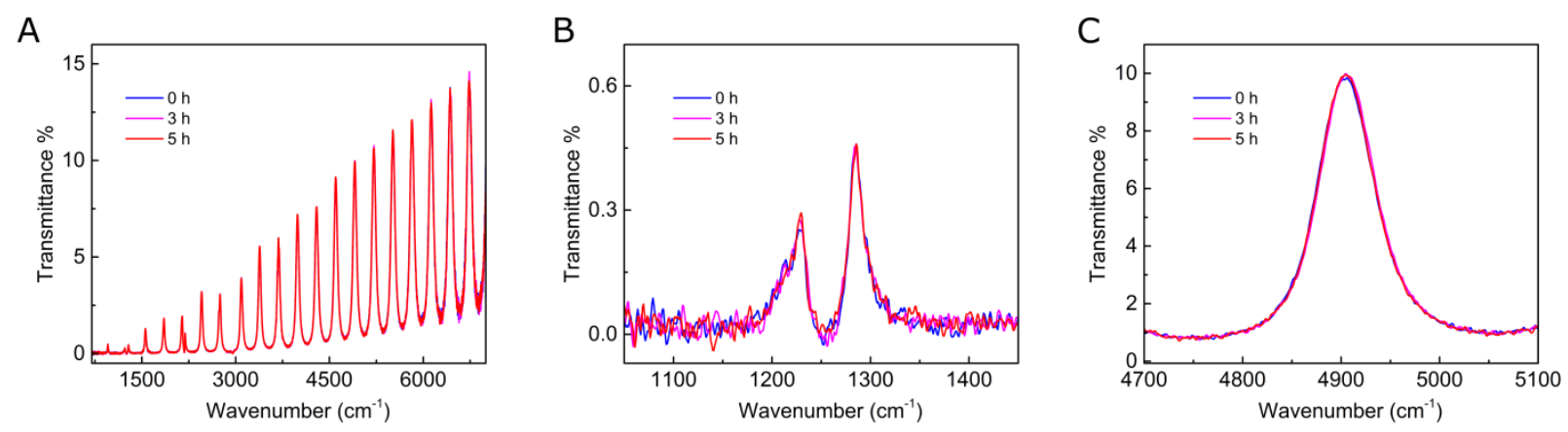

Fig. S5. FT-IR transmission spectrum of strongly coupled 1 in the total spectral range of 500-7000 $\mathrm{cm}^{-1}(\mathrm{~A})$, the zoomed region of the strongly coupled vibrational mode (B), and the higher order 
cavity mode (C). The spectrum was recorded immediately after injection of 1 (blue curve), after $3 \mathrm{~h}$ (magenta curve) and after 5h (red curve).

\section{FT-IR transmission spectrum of an off-resonance cavity}

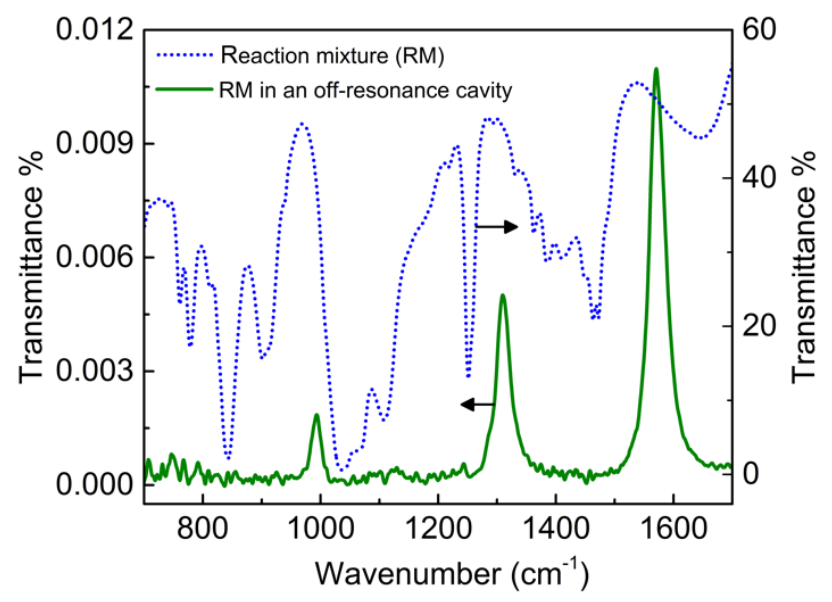

10 Fig. S6. FT-IR transmission spectrum of the reaction mixture injected to into an off-resonance cavity (green curve), and the spectrum recorded outside the cavity (blue dotted line). No vibrational modes of interest are strongly coupled.

\section{Products identification and quantitative GC-MS analysis}

Reaction products 1 and $\mathbf{2}$ were identified by comparing the mass spectra and retention times against commercially available samples of $\mathbf{1}$ and $\mathbf{2}$ (Fig. S7). The GC chromatogram is given in the top panel of Figs. S7 A and B and the respective mass fragments are given in bottom panels. The mass spectrum given in magenta colour in Fig S7A corresponds to the second peak of the chromatogram (retention time $2.42 \mathrm{~min}$ ) and the mass spectra given in orange colour corresponds to the first peak (retention time $2.29 \mathrm{~min}$ ). The first peak in GC chromatogram of Fig. S7A is coming from the decomposition of $\mathbf{1}$ on the GC column. The GC chromatogram and the corresponding mass fragments of 2 are shown in Fig. S7B. 


\section{Science}

MIAAAS

A
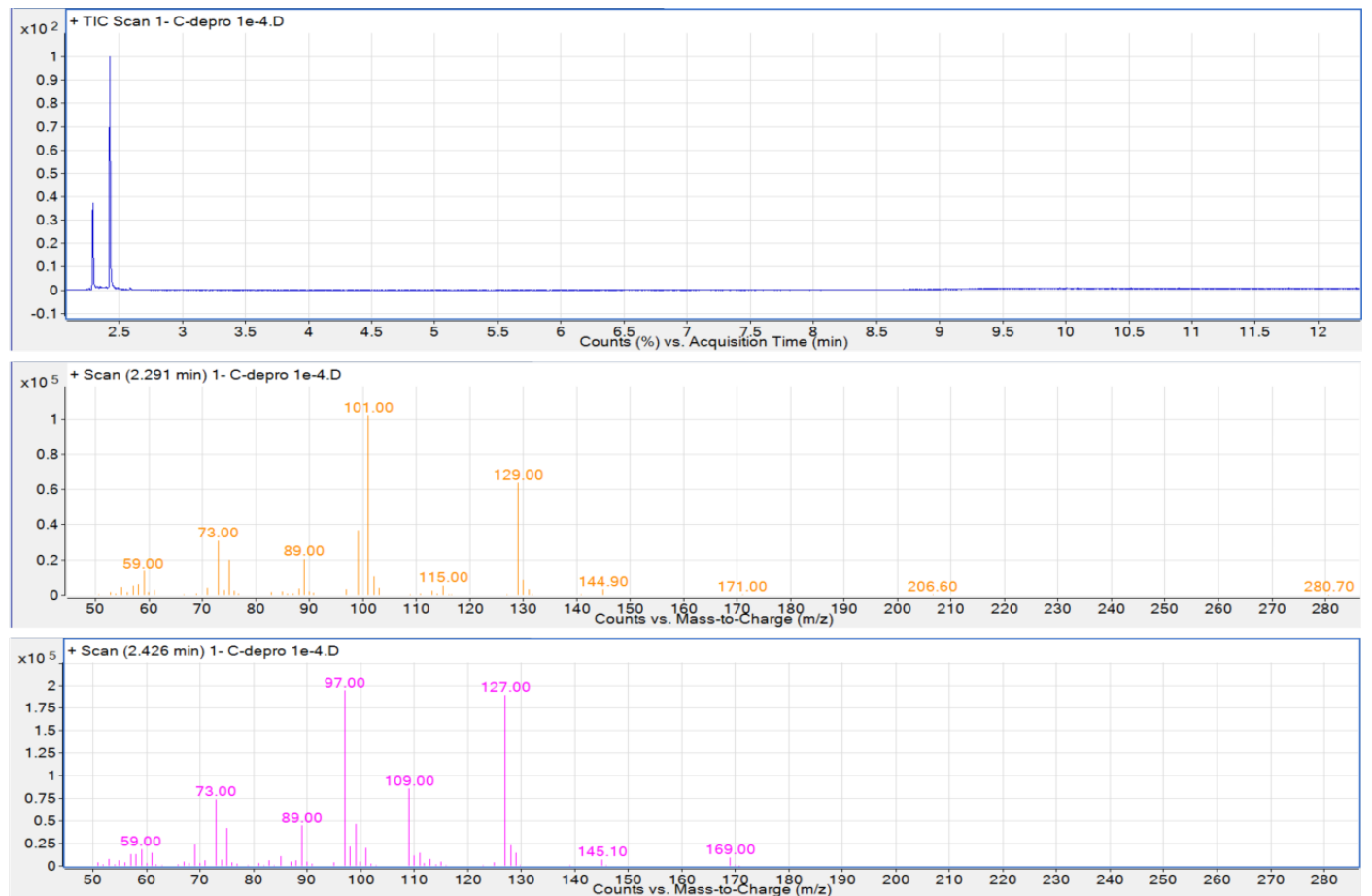

B
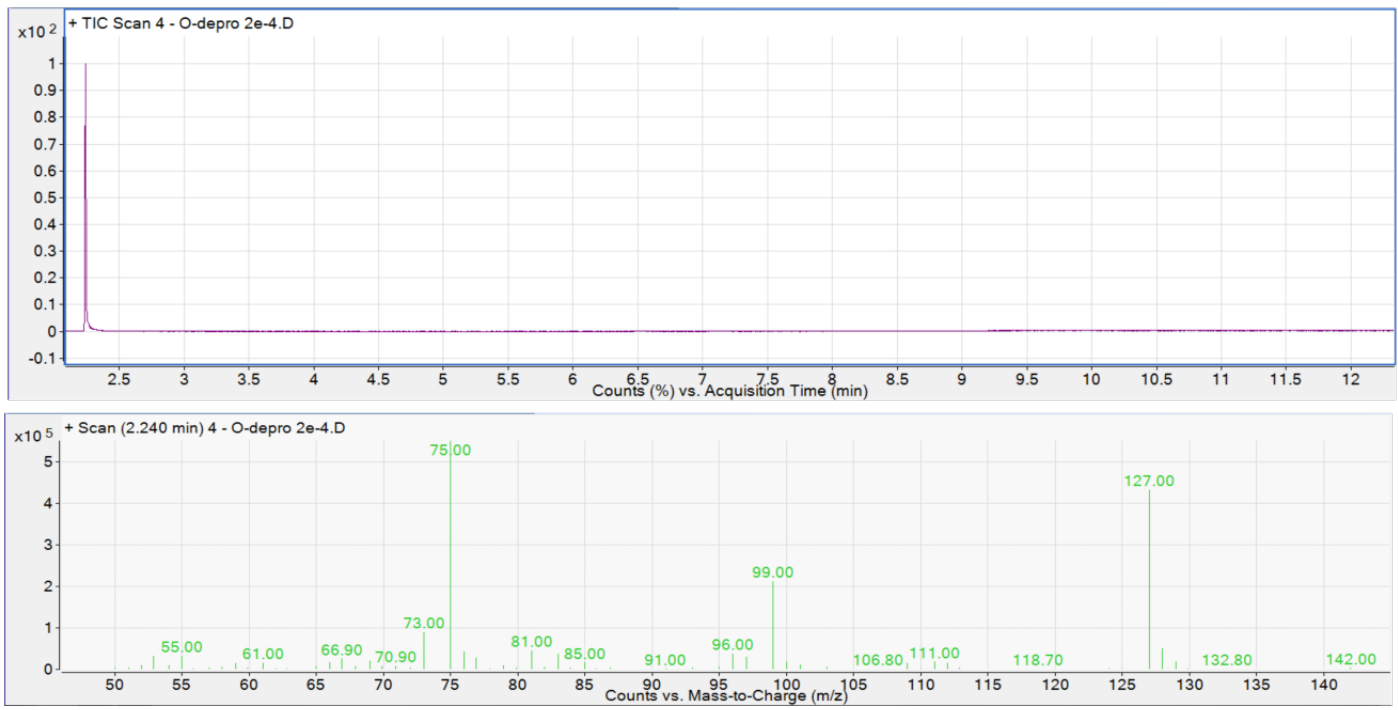

Fig. S7. GC chromatogram (top panel) and mass spectra (bottom panels) of commercially available product $\mathbf{1}$ (A) and $\mathbf{2}$ (B). The concentration of the samples 1 and $\mathbf{2}$ used for this analysis was 0.1 $\mathrm{mM}$ and $0.2 \mathrm{mM}$, respectively. 


\section{GC-MS calibration curves and the analysis of the products}

$200 \mu \mathrm{L}$ solutions of compound 1 and 2 at different concentrations ( 0.1 to $10 \mathrm{mM})$ were prepared by diluting stock solutions of these compounds in isopropanol. For each compound, a six-point graph was plotted; correlating the characteristic GC peaks (as integrated automatically by the Agilent MassHunter Workstation v.B.06.00 software) with substrate concentration (Fig. S8). Each data point was the mean of three independent measurements and the correlation line was obtained from the least-squares fitting (intercept $=0$ ). Error bars on graphs are shown as \pm standard deviation for each data point.

A

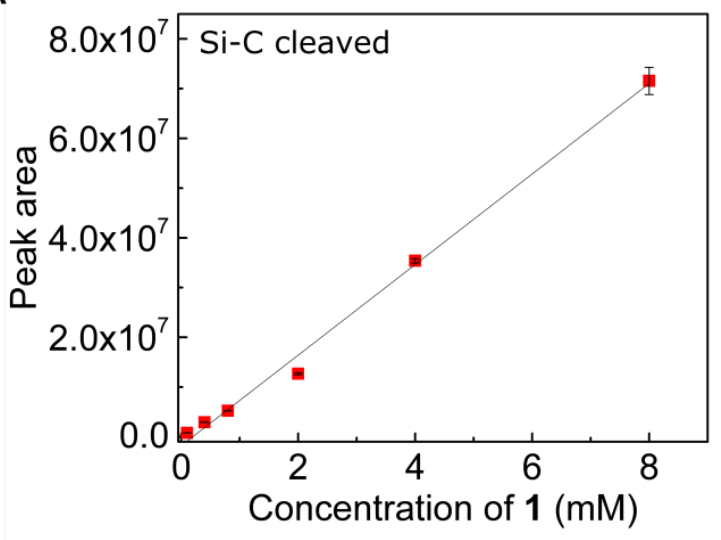

B

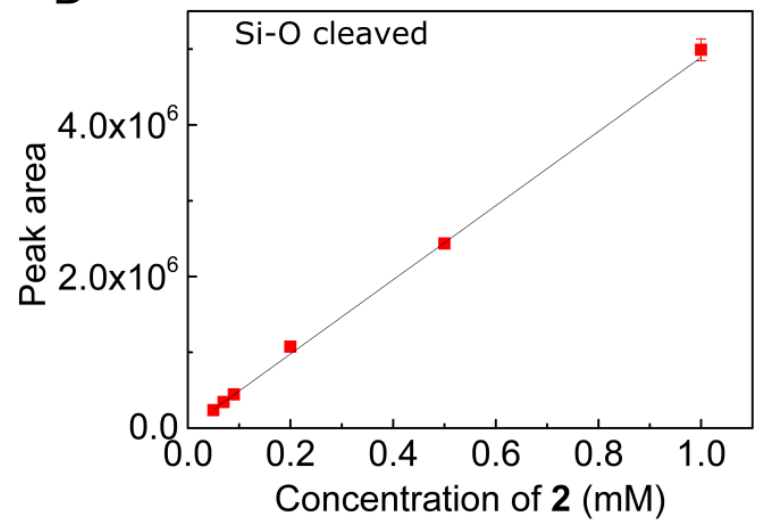

Fig. S8. Correlation between the concentration of the products in isopropanol and the measured gas chromatography peak areas. The calibration curve for $\mathrm{C}$ - and O-deprotected products are shown in (A) and (B) respectively.

After $3 \mathrm{~h}$ of reaction, the microfluidic Fabry-Pérot cavity (for on- and off-resonance measurements) as well as the liquid cells of the outside the cavity experiments was opened and the reaction mixture was extracted with isopropanol $(1 \mathrm{~mL}) .200 \mu \mathrm{L}$ of this solution were directly injected in GC-MS. The concentrations of $\mathbf{1}$ and $\mathbf{2}$ in the reaction mixture were calculated by comparing the product peak area with the calibration line and the ratio between the two products (1 over 2) was determined. The reported ratios are an average of multiple runs, with an error corresponding to the mean $( \pm)$ absolute deviation. 


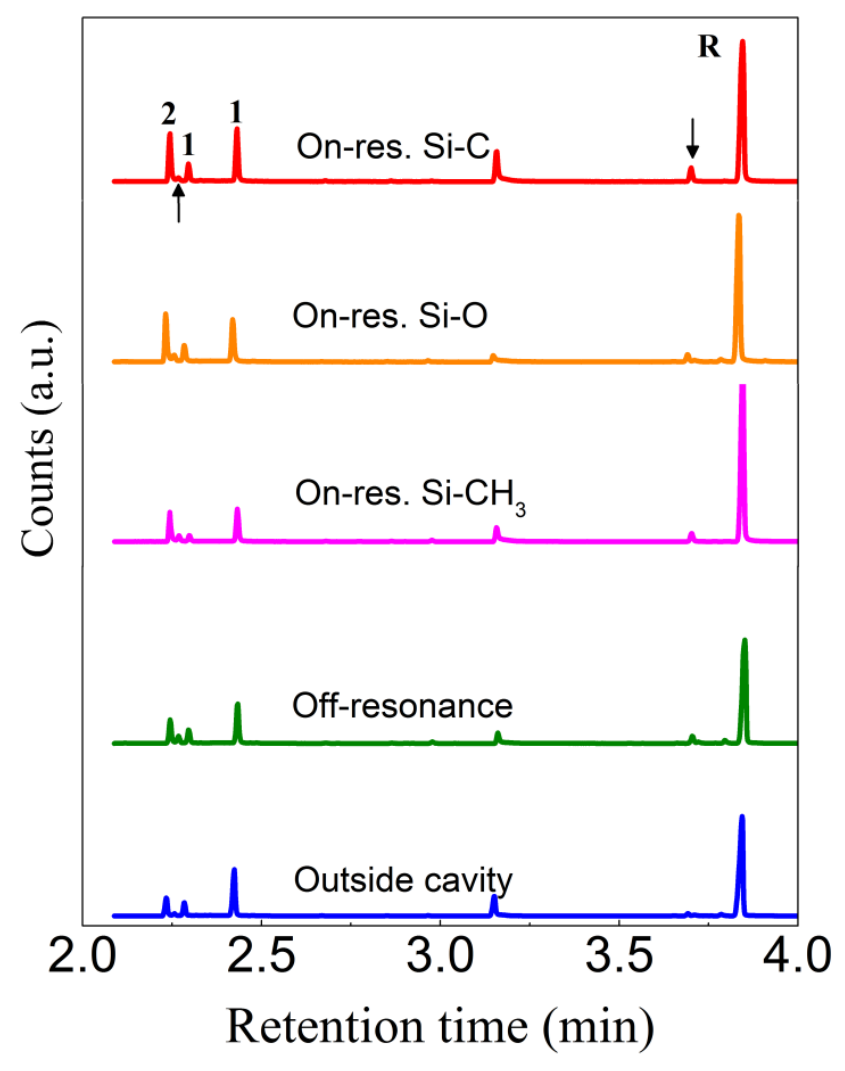

Fig. S9. The GC-MS chromatograms of the reactions carried out in cavities tuned to be onresonance with $\mathrm{Si}-\mathrm{C}$ stretching (red), Si-O stretching (orange) and $\mathrm{Si}-\mathrm{CH}_{3}$ bending modes (magenta), together with the data for an off-resonance cavity (green) and outside the cavity (blue). The unreacted starting material is marked as $\mathbf{R}$. The peaks corresponding to $\mathrm{C}$ - and $\mathrm{O}$ - deprotected products are marked as $\mathbf{1}$ and $\mathbf{2}$, respectively. The peaks indicated by the arrows are impurities that come from the slow degradation of the GC-MS column and it is present in all the chromatograms that we measure. The peak at the retention time of 3.2 min corresponds to the TBAF which is eluted as an ion pair in the GC column.

As explained in the main text, the ratio of the yields of the two products on resonance has to be corrected for the area under VSC which corresponds to ca. $60 \%$ of the FP microfluidic cell (as explained above). The other $40 \%$ is detuned beyond the width of the vibrational mode being coupled and hence is in the off-resonance condition. Table S1 below shows the uncorrected and corrected ratios of products 1 and 2. 
Table S1: Table displaying the observed product ratios ([1]/[2]) from GC-MS measurements and the corresponding corrected values under VSC for the tuned area.

\begin{tabular}{|c|c|c|c|}
\hline \multirow{2}{*}{\multicolumn{2}{|c|}{ Experiment }} & \multicolumn{2}{|c|}{$\begin{array}{l}\text { Product ratio } \\
([1] /[2])\end{array}$} \\
\hline & & Observed & $\begin{array}{c}\text { Corrected } \\
\text { for tuned } \\
\text { area } \\
\text { under } \\
\text { VSC }\end{array}$ \\
\hline \multicolumn{2}{|c|}{$\begin{array}{l}\text { Outside } \\
\text { cavity }\end{array}$} & $1.5 \pm 0.2$ & \\
\hline \multicolumn{2}{|c|}{$\begin{array}{l}\text { Off- } \\
\text { resonance }\end{array}$} & $1.4 \pm 0.1$ & \\
\hline \multirow[t]{4}{*}{ VSC } & $\mathrm{Si}-\mathrm{C}$ & $0.8 \pm 0.1$ & $0.3 \pm 0.1$ \\
\hline & $\mathrm{Si}-\mathrm{O}$ & $0.9 \pm 0.1$ & $0.4 \pm 0.1$ \\
\hline & $\mathrm{Si}-$ & $0.8 \pm 0.1$ & $0.3 \pm 0.1$ \\
\hline & $\mathrm{CH}_{3}$ & & \\
\hline
\end{tabular}



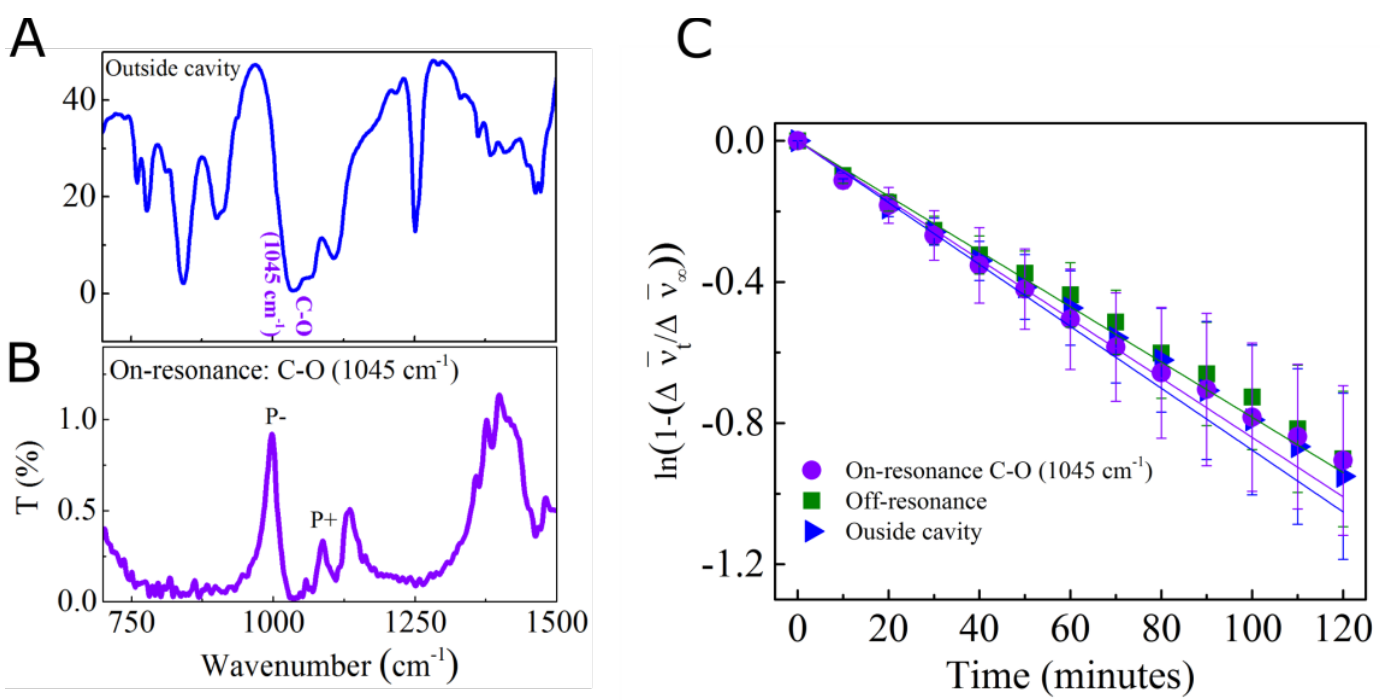

Fig. S10. (A) FT-IR transmission spectrum of the starting material $\mathbf{R}$ in the reaction mixture consisting of $\mathrm{MeOH}$ and THF, recorded outside cavity with the $\mathrm{C}-\mathrm{O}$ stretching vibration noted in the plot. (B) The FT-IR transmission spectra showing the vibrational strong coupling of the C-O stretching vibration of $\mathbf{R}, \mathrm{MeOH}$ and THF. (C) Kinetics of the reactions in cavities tuned to be onresonance with the $\mathrm{C}-\mathrm{O}$ stretching mode of $\mathbf{R}$ (violet circles) together with an off-resonance cavity (green squares) and outside the cavity (blue triangles).

\section{Reference}

46. A. D. Rakić, A. B. Djurišić, J. M. Elazar, M. L. Majewski, Appl. Opt., AO. 37, 5271-5283 (1998). 
Science

5

10

15

20

25

30

35 